\title{
Amiodarone-induced pulmonary toxicity mimicking metastatic lung disease
}

\author{
P. Patel, D. Honeybourne and R.D.S. Watson
}

Departments of Respiratory Medicine and Cardiology, Dudley Road Hospital, Birmingham B18 7QH, UK.

\begin{abstract}
Summary: A 65 year old man developed atrial arrhythmias secondary to a congestive cardiomyopathy which were resistant to quinidine and disopyramide. Amiodarone controlled the paroxysmal atrial tachycardia but 4 months after starting the drug he developed increasing dyspnoea and radiological changes highly suggestive of metastatic lung disease. Lung biopsy showed change of drug-induced pneumonitis and 4 months after stopping amiodarone his symptoms resolved and the chest $\mathrm{X}$-ray had cleared. Amiodarone may cause pulmonary toxicity mimicking metastatic lung disease.
\end{abstract}

\section{Introduction}

Amiodarone has been reported to produce a variety of radiological abnormalities and the following case report describes a further change due to lung damage caused by this drug.

\section{Case report}

A 65 year old man presented with palpitations and dizziness. Clinical examination was normal. Chest Xray, ambulatory electrocardiogram (ECG) recordings, full blood count, blood profile and thyroid function tests were all normal. Two months later he presented with a history of severe dyspnoea, and on examination he had a regular tachycardia of 150 beats/min with evidence of left ventricular failure. His ECG showed atrial tachycardia with a 2:1 atrioventricular block. Chest X-ray at that time showed left ventricular failure but no other abnormality. An echocardiogram showed global impairment of left ventricular function suggestive of congestive cardiomyopathy. He was initially treated with digoxin, diuretics and warfarin but the atrial tachycardia persisted and eventually required cardioversion.

Both quinidine $(500 \mathrm{mg} /$ day $)$ and disopyramide $(500 \mathrm{mg} /$ day $)$ failed to prevent paroxysmal atrial tachycardia; amiodarone $(400 \mathrm{mg} /$ day) successfully prevented paroxysms.

Four months later he complained of increasing dyspnoea on effort. There was no history of chest pain

Correspondence: D. Honeybourne M.D., M.R.C.P., Department of Respiratory Medicine, Dudley Road Hospital, Birmingham B18 7QH, UK.

Accepted: 3 December 1986 or weight loss. On examination his chest was clear. Investigations, including full blood count, ESR, liver function tests, rheumatoid factor, auto-antibodies and acid phosphatase, showed no abnormality. However, his chest X-ray (Figure 1) showed several discrete round shadows in the right mid and lower zones, varying from 0.5 to $2 \mathrm{~cm}$ in diameter, suggestive of metastatic lung disease. The left lung was entirely normal. Lung function tests showed normal volumes and carbon monoxide gas transfer. However, the arterial $\mathrm{PO}_{2}$ was reduced to $9.2 \mathrm{kPa}(69 \mathrm{mmHg})$. Fibreoptic bronchoscopy was performed which showed no endobronchial abnormality but transbronchial biopsies from the right lower lobe showed accumulation of foamy macrophages in the alveolar spaces, hyperplasia of type II pneumocytes and widening of the alveolar septae confirming typical changes associated with drug-induced pneumonitis. No malignant cells were seen at histology and cytology was negative.

The serum amiodarone level was $1.9 \mathrm{mg} / 1$. Amiodarone was discontinued but the other medications remained unchanged and his dyspnoea resolved. A chest X-ray 4 months later showed complete resolution of the opacities (Figure 2). Clinically he has remained asymptomatic and has not required steroid therapy.

\section{Discussion}

The pulmonary side effects from amiodarone may occur after a few weeks, or even several years, following the onset of treatment. ' The effect is initially that of a pneumonitis which may progress to pulmon- 


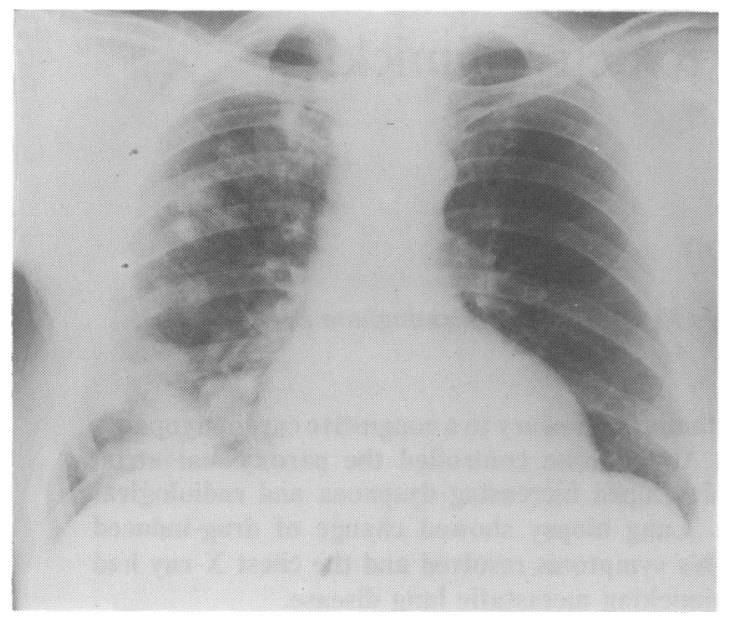

Figure 1 Chest X-ray four months after starting amiodarone therapy.

ary fibrosis, and histology usually shows evidence of diffuse alveolar damage. The usual chest X-ray appearance is that of bilateral diffuse interstitial and patchy peripheral alveolar infiltration frequently affecting the upper lobes. ${ }^{2}$ Rarely, pleural thickening and effusions have been described. ${ }^{3}$

Our case is unique in that the radiological appearance showed only unilateral changes which were very discrete. The finding of a normal carbon monoxide transfer test was also unusual as lung involvement is usually diffuse.

Both immunological ${ }^{4}$ and direct toxic effects ${ }^{3}$ have

\section{References}

1. McGovern, G., Garan, H., Kelly, E. \& Ruskin, J.N. Adverse reactions observed during treatment with amiodarone hydrochloride. $\mathrm{Br} \mathrm{Med} J$ 1983, 287: 175180.

2. Marchlinski, F.E., Gansler, T.S., Waxman, H.L. \& Josephson, M.E. Amiodarone pulmonary toxicity. Ann Int Med 1982, 97: 829-845.

3. Darmanata, J.I., von Zandwijk, N., Duren, D.R. et al. Amiodarone pneumonitis: three further cases with a review of published reports. Thorax 1984, 39: 57-64.

4. Suarez, L.D., Poderoso, J.J., Elsner, B., Bunster, A.M., Esteva, H. \& Bellotti, M. Subacute pneumopathy during

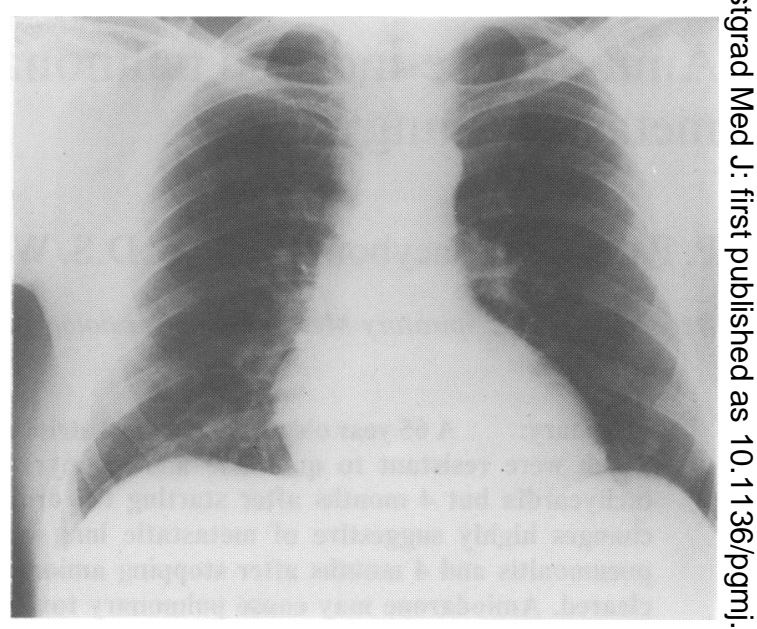

Figure 2 Chest X-ray four months after discontinuing amiodarone.

been implicated as mechanisms for this disorder. The incidence of pulmonary toxicity is probably lower if the dose of amiodarone is limited to no more than $400 \mathrm{mg} / \mathrm{day},{ }^{5}$ although this was never exceeded in our patient. Resolution usually occurs following withdrawal of amiodarone but fatalities have been reporteo. e.g. 9 out of 39 patients in one series. ${ }^{6}$

Amiodarone has been reported as causing chest $X$ ray appearances mimicking tuberculosis, ${ }^{7}$ but our case seems to be the first reported to show changes resembling metastatic lung disease.

amiodarone therapy. Chest 1983, 83: 566-568.

5. Rotmensch, H.H., Livon, M., Tupilski, M. \& Laniado, S. Possible association of pneumonitis with amiodarone therapy. Am Heart J 1980, 100: 412-413.

6. Rakita, L., Sobol, S.M., Mostow, N. \& Vrobel, T. Amiodarone pulmonary toxicity. Am Heart J 1983, 106: 906-916.

7. Zahker, C., Hamer, A., Peter, T. \& Mandel, W. Low- O dose steroid therapy for prophylaxis of amiodarone- $N$ induced pulmonary infiltrates. $N$ Engl J Med 1983, 308: 779. 